\title{
How many challenges we may encounter in anterior megalophthalmos with white cataract: a case report
}

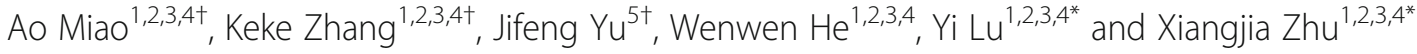

\begin{abstract}
Background: Anterior megalophthalmos is a rare congenital disease which mainly features enlargement of the anterior segment. Cataract surgeries in anterior megalophthalmos can be challenging due to the anatomical anomalies while the studies upon the surgical design have been less integrated.

Case presentation: A 37-year-old woman presented with progressively blurred vision in the right eye after a transient fever 10 months ago. Her ocular history included hypermetropia with a spherical equivalent of $+4.00 \mathrm{OU}$. The review of systems showed bilateral varus deformity of distal interphalangeal joints on the little fingers. The patient denied family history of hereditary ocular diseases and her sister was born with uterus didelphys. On initial examinations, the corrected distance visual acuity was hand motion OD and 20/33 OS. Her intraocular pressure was $15 \mathrm{mmHg} O D$ and $16 \mathrm{mmHg}$ OS. Horizontal corneal diameter was $14 \mathrm{~mm}$ OD and $13.88 \mathrm{~mm}$ OS and axial length was $24.87 \mathrm{~mm}$ OD and $25 \mathrm{~mm}$ OS. Anterior segment photography showed bilateral iridal atrophy with deficiency in pupillary dilation and white cortically mature cataract in the right eye. Inspection by anterior segment optical coherence tomography indicated bilateral augmented anterior chambers with backward iridal concave on horizontal orientation. Ultrasound biomicroscopy showed partially peripheral anterior synechiae and pectinate ligaments at chamber angle in both eyes and opacified lens with the apparently elongated suspensory ligaments in the right eye. A deliberately selected 1-piece foldable intraocular lens (IOL) with frame haptics was implanted after phacoemulsification for good IOL stability. During the follow-up, the visual rehabilitation appeared relatively good and a lower degree of IOL dislocation comparing with existing reports was verified by OPD-Scan III aberrometry.

Conclusions: We presented the challenges and the original findings from a case of congenital anterior megalophthalmos with white cataract who underwent phacoemulsification and IOL implantation. This is the first report describing the comparison of the different IOL power calculation formulas in anterior megalophthalmos. Compared to the SRKT and the Holladay II formulas, the Haigis formula could be a more accurate choice for the IOL calculation in anterior megalophthalmos according to our case. Moreover, the deliberate selection of IOLs is essential for IOL stability in these patients.
\end{abstract}

Keywords: Anterior megalophthalmos, Cataract, Intraocular lens, Dislocation, IOL power calculation, Haigis formula

\footnotetext{
*Correspondence: luyieent@126.com; zhuxiangjia1982@126.com

${ }^{\dagger}$ Ao Miao, Keke Zhang and Jifeng Yu contributed equally to this work.

${ }^{1}$ Eye Institute, Eye and ENT Hospital, Shanghai Medical College, Fudan

University, Shanghai 200031, China

Full list of author information is available at the end of the article
}

(c) The Author(s). 2019 Open Access This article is distributed under the terms of the Creative Commons Attribution 4.0 International License (http://creativecommons.org/licenses/by/4.0/), which permits unrestricted use, distribution, and reproduction in any medium, provided you give appropriate credit to the original author(s) and the source, provide a link to the Creative Commons license, and indicate if changes were made. The Creative Commons Public Domain Dedication waiver (http://creativecommons.org/publicdomain/zero/1.0/) applies to the data made available in this article, unless otherwise stated. 


\section{Background}

Anterior megalophthalmos is a rare congenital disease which mainly features bilateral megalocornea, broad deep anterior chambers and ciliary ring enlargement with quite normal axial length (AL) [1-3]. The pathogenesis relates mostly to hereditary factors while the mechanism remains unclear. Since the congenital lesions of anterior megalophthalmos are always nonprogressively asymptotic and easy to be overlooked, onset of cataract and lens subluxation are the most common initial symptoms of this disease. Other complications may include anterior embryotoxon, mosaic corneal dystrophy, iris atrophy, spontaneous vitreous hemorrhage, retinal detachment, and peripheral retinal degenerations [4-6].

Cataract surgeries on patients with anterior megalophthalmos are challenging. Deficiency of pupillary dilation can always be observed due to iris atrophy [1]. Zonular anomalies and enlarged sulcus will also highly increase the risk of postoperative intraocular lens (IOL) dislocation at the same time [7]. For the anatomical anomalies of anterior megalophthalmos, deliberate selection of IOLs and power calculation formulas before the cataract surgery are vital for small postoperative refractive error and matter a lot to visual rehabilitation. In addition, a more careful follow-up is necessary for patients with anterior megalophthalmos to prevent postoperative complications [8].

We report a challenging case of anterior megalophthalmos accompanied with secondary white cataract (cortically mature) who underwent phacoemulsification and in-the-bag IOL implantation successfully, highlighting the rather good visual rehabilitation by deliberate selection of IOLs and postoperative follow-up.

\section{Case presentation}

A 37-year-old woman presented to our hospital with complaints of blurred vision in the right eye for 10 months. She had no family history of hereditary ocular diseases and no previous history of eye surgery or ocular trauma. In addition, the patient had bilateral varus deformity of distal interphalangeal joints on the little fingers. Also, her sister was born with uterus didelphys.

\section{Preoperative examinations \\ Routine inspections}

The best corrected visual acuity (BCVA) was hand motion in the right eye and $20 / 33$ in the left eye. The refraction was $+4.25 /-0.50 \times 90$ in the left eye. Horizontal corneal diameters were apparently enlarged in both eyes (the right eye $14 \mathrm{~mm} /$ the left eye $13.88 \mathrm{~mm}$ ). Applanation intraocular pressure (IOP) were normal in both eyes. Main measures of the present case are summarized in Table 1.
Table 1 Main preoperative measures of the present case

\begin{tabular}{lll}
\hline Quantitative measurements & OD & OS \\
\hline AL & $24.87 \mathrm{~mm}$ & $25.00 \mathrm{~mm}$ \\
LT & $5.23 \mathrm{~mm}$ & $3.60 \mathrm{~mm}$ \\
ACD & $2.644 \mathrm{~mm}$ & $3.677 \mathrm{~mm}$ \\
WTW & $14.00 \mathrm{~mm}$ & $13.88 \mathrm{~mm}$ \\
Chamber Angle & $36.0^{\circ}$ & $76.8^{\circ}$ \\
CCT & $535 \mathrm{um}$ & $537 \mathrm{um}$ \\
K1/K2 & $39.2 \mathrm{D} / 40.3 \mathrm{D}$ & $39.1 \mathrm{D} / 39.8 \mathrm{D}$ \\
Applanation IOP & $15 \mathrm{mmHg}$ & $16 \mathrm{mmHg}$ \\
Count of corneal endothelium & $2544 / \mathrm{mm}^{2}$ & $2821 / \mathrm{mm}^{2}$ \\
\hline
\end{tabular}

$A L$ axial length, $L T$ lens thickness, $A C D$ anterior chamber depth, WTW white to white, $C C T$ central corneal thickness, IOP intraocular pressure

\section{Special examinations}

Anterior segment photography showed mild iridal atrophy of both eyes, which subsequently lead to insufficiently dilated pupils with diameters no more than 5 $\mathrm{mm}$. White cataract was observed in the right eye (Fig. 1) . Inspection by anterior segment optical coherence tomography (AS-OCT) (Cornea/Anterior Segment OCT SS1000, Tomey Corporation, Japan) indicated bilateral augmented anterior chambers with backward iridal concavity on horizontal orientation, although the backward concavity in the right eye was markedly reduced before the surgery because of the swelling cataractous lens; while on vertical orientation iris revealed rather flat (Fig. 2a-d). Ultrasound biomicroscopy (UBM) (MEDA $M D-300 L$ ) showed opacified lens with the apparently elongated suspensory ligaments in the right eye. Partially peripheral anterior synechiae and pectinate ligaments at anterior chamber angle were also observed in both eyes. Ciliary processes were small and scleral processes were not apparent under UBM inspection (Fig. 3a-b).

On the basis of the above findings, the patient was diagnosed with bilateral anterior megalophthalmos complicating white cataract in the right eye.

\section{Surgical procedure}

The operation was performed by an experienced surgeon (Y.L.). A $2.6 \mathrm{~mm}$ temporal clear corneal incision was made under topical anesthesia. Viscoelastic agent (DisCoVisc, Alcon, Fort Worth, TX, USA) was then instilled to maintain the anterior chamber with small pupil. Following a continuous curvilinear capsulorhexis of $5.5 \mathrm{~mm}$ in size, hydrodissection, chopping, nucleus rotation, and phacoemulsification (CENTURION Vision System, Alcon, Fort Worth, TX, USA) were then performed. A 1-piece foldable IOL $(+20.5 \mathrm{D}$, Human Optics PC Acrylic IOL, MC X11 ASP) with four frame haptics to increase intracapsular stability, was inserted into the capsular bag. After aspiration of residual viscoelastic, the incision was hydrated with balanced salt solution and 


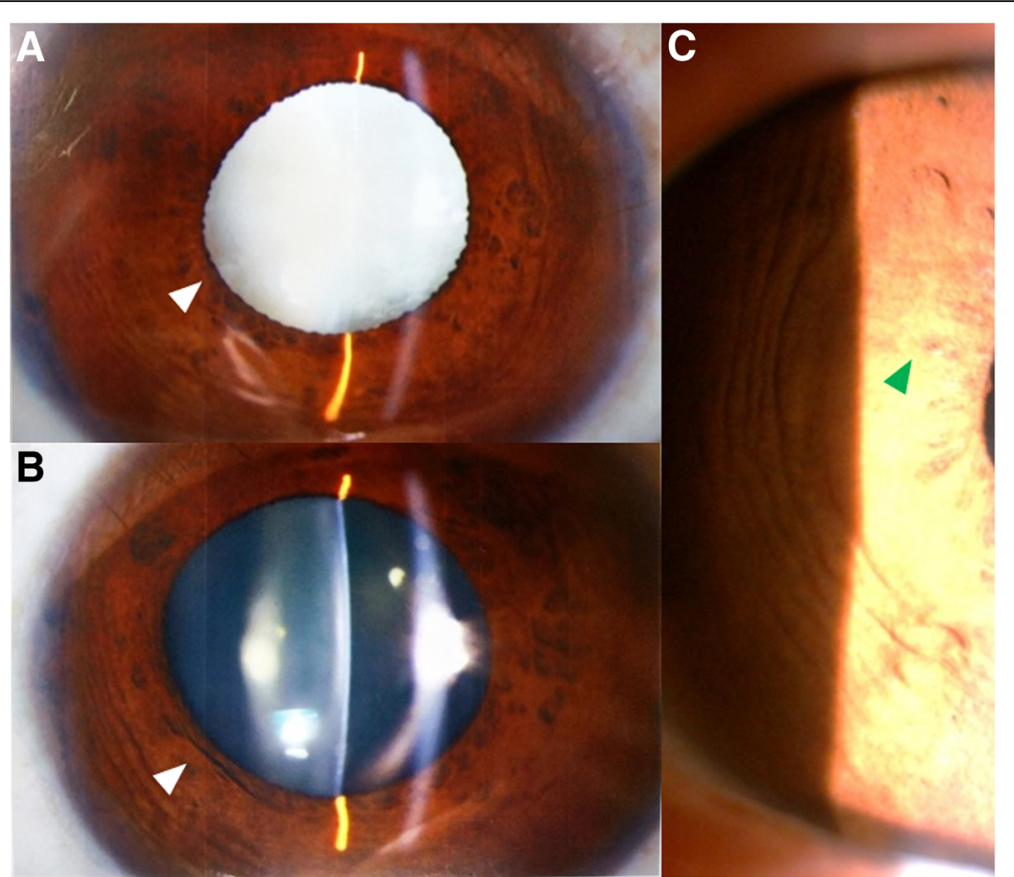

Fig. 1 Preoperative slit-lamp examination. a, b Preoperative slit-lamp examination of the right eye and the left eye showing enlarged corneas, deep anterior chambers, insufficiently dilated pupils (indicated with white arrowheads) and white cataract in the right eye. c Preoperative slitlamp photography of the right eye showing mild concomitant iridal atrophy (indicated with green arrowhead)

checked for water tightness. Gentle operation was emphasized intraoperatively considering zonular weakness and the deep anterior chamber as the infusion bottle height was set to $75 \mathrm{~cm}$ and the phacoemulsification was carried out in a slow-motion mode (vacuum: $300 \mathrm{mmHg}$; aspiration flow rate: $28 \mathrm{cc} / \mathrm{min}$ ). Postoperatively, Cravit Eye Drops (Alcon Laboratories, Inc., Fort Worth, TX, USA), Pred Forte Eye Drops (Allergan Pharmaceuticals, Inc., Dublin, Ireland), and Diclofenac Sodium Eye Drops (Shenyang Xingqi Pharmaceutical Co. Ltd, Shenyang, China), all 3 times a day for 4 weeks, were given.

\section{Postoperative follow-up}

One month after the surgery, the uncorrected visual acuity (UCVA) and BCVA of the right eye improved to $20 / 25$ and $20 / 20$. The actual postoperative refraction was $+1.50 /-0.50 \times 115$ in the right eye and the actual postoperative spherical equivalent (SE) was +1.25 D. The IOP of the right eye was $18 \mathrm{mmHg}$. Postoperative inspection by AS-OCT indicated a low degree of IOL decentration and iridal backward concavity still remained on horizontal orientation with flat iris on vertical orientation (Fig. 2e, f). OPD-Scan III aberrometry (Nidek Co, Ltd, Gamagori, Japan) verified increased internal coma and tilt aberrations indicating slight dislocation of IOL after surgery (Table 2, Fig. 4). Three months after the surgery, the visual acuity and the refractive status of the operated eye were stable and the IOP remained within normal range $(15.0 \mathrm{mmHg}$ OD and $17.8 \mathrm{mmHg}$ OS). The IOL also showed good centering and stability during the follow-up.

The postoperative refractive error equals to the actual postoperative SE $(+1.25 \mathrm{D})$ minus the predicted refraction calculated by IOL power calculation formulas as following:

\section{Postoperative Refractive Error $=$ Actual Postoperative SE - Predicted Refraction;}

In this case, we originally used the SRK/T formula for IOL power calculation and the postoperative refractive error turned out to be $+1.44 \mathrm{D}$ during the follow-up. To find the most accurate power calculation formula with lowest postoperative refractive error for the challenging anterior megalophthalmos cases, we further applied the Haigis and Holladay II formulas (Table 3).

The postoperative refractive error decreased to a rather low value as $-0.05 \mathrm{D}$ after using the Haigis formula. As to the Holladay II formula, the postoperative refractive error was $+0.63 \mathrm{D}$. Therefore, compared to the SRK/T and the Holladay II formulas, the Haigis formula may be a more suitable choice according to our case, with higher accuracy and lower postoperative refractive error concerning to the IOL calculation in anterior megalophthalmos. 

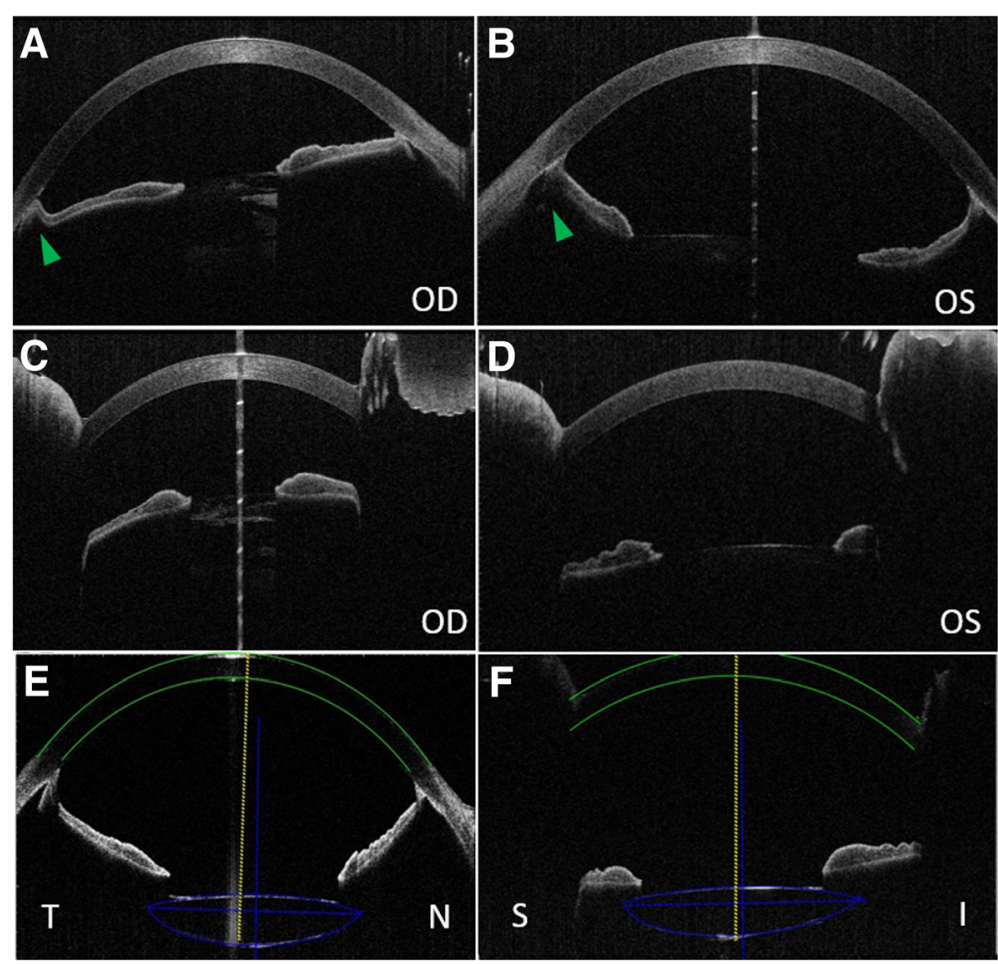

Fig. 2 Preoperative and postoperative anterior segment optical coherence tomography (AS-OCT). a, b Preoperative AS-OCT on horizontal orientation verifying augmented anterior chambers in both eyes with backward iridal concavity in the left eye and markedly reduced backward iridal concavity resulted from the swelling cataractous lens in the right eye (indicated with green arrowheads). c, $\mathbf{d}$ Preoperative AS-OCT on vertical orientation of the right eye and the left eye verifying augmented anterior chambers with rather flat iris. e Postoperative AS-OCT inspection of the right eye on horizontal orientation showing slight decentration of IOL and backward iridal concavity. $\mathbf{f}$ Postoperative AS-OCT inspection of the right eye on vertical orientation showing slight decentration of IOL and flat iris

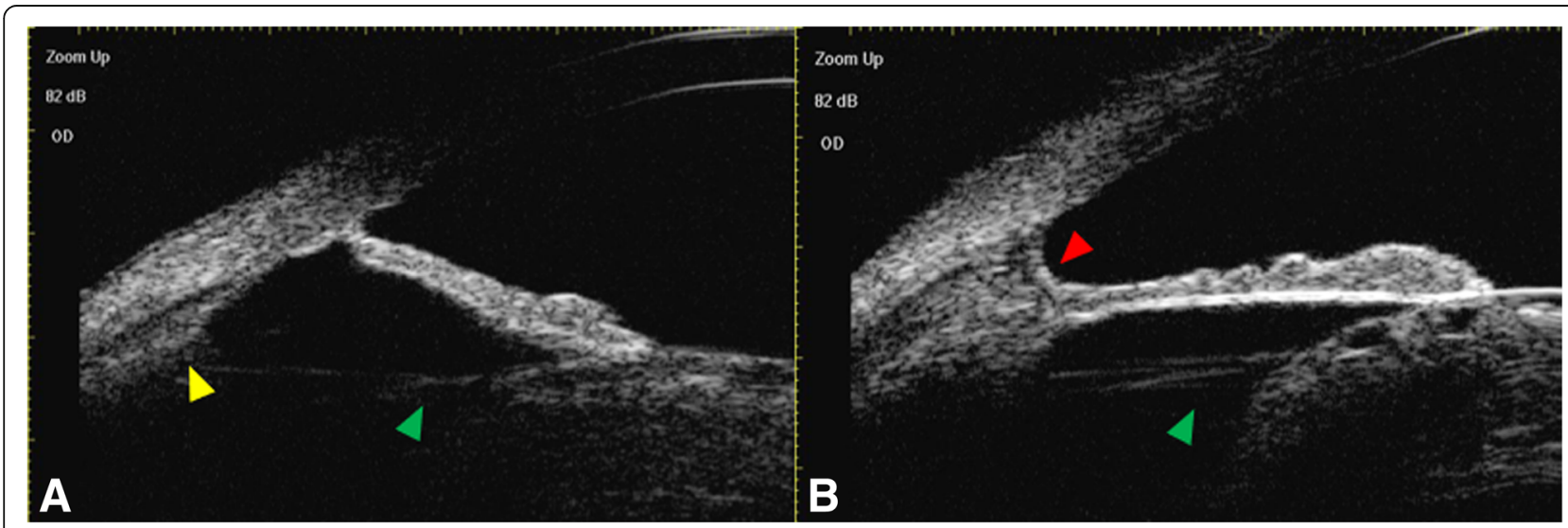

Fig. 3 a, b Preoperative ultrasound biomicroscopy of the right eye. Preoperative ultrasound biomicroscopy of the right eye showing the cloudy cataractous lens suspended to the small ciliary processes (indicated with yellow arrowhead) by the apparently elongated suspensory ligaments (indicated with green arrowheads) caused by congenital enlargement of the anterior segment. The pectinate ligament (indicated with red arrowhead) was also observed at some places of anterior chamber angle by ultrasound biomicroscopy 
Table 2 Postoperative OPD-Scan III aberrometry results of the right eye

\begin{tabular}{llll}
\hline & Zernike/OPD & Zernike/Corn & Zernike/Int \\
\hline Tilt & $0.958 @ 342$ & $0.234 @ 234$ & $1.058 @ 355$ \\
High & 0.467 & 0.337 & 0.514 \\
T. Coma & 0.365 & 0.156 & 0.383 \\
T. Trefoil & 0.256 & 0.130 & 0.166 \\
T. Sph & $0.041(\mathrm{C} 12-0.039)$ & $0.237(\mathrm{C} 12+0.236)$ & $0.283(\mathrm{C} 12-0.282)$ \\
\hline
\end{tabular}

\section{Genetic examination}

For genetic analysis, we obtained the blood samples of the patient, her son and her parents for whole-exome sequencing based on family to identify any gene mutations directly related to anterior megalophthalmos. However, no known or newfound related gene mutations were found (Table 4).

\section{Discussion and conclusions}

In this challenging case, we successfully performed the phacoemulsification and IOL implantation for a congenital anterior megalophthalmos patient with white cataract. Postoperative examinations showed relatively good visual outcomes with a lower degree of IOL dislocation comparing with existing reports. This is also the first report comparing the different IOL power calculation formulas in anterior megalophthalmos.

All the clinical phenotypes in this case were in accordance with the previously reported diagnostic criteria of anterior megalophthalmos, including bilaterally enlarged corneal diameter (more than $13 \mathrm{~mm}$ ), deepened anterior chambers, elongated ciliary ring, atrophic iris, overstrained suspensory ligaments, and premature cataract [9]. In addition, corneal thickness, IOP and AL are supposed to be roughly normal. As to genetic factors, $50 \%$ of this congenital disease transmits with $\mathrm{X}$-linked recessive inheritance and men consequently constitute most of the patients [10]. We suppose that the present case may follow some other hereditary pattern, which means, it could be resulted from autosomal transmission as the patient was a female. Therefore, we diagnosed the patient as anterior megalophthalmos.

During routine clinical practice, we should also pay attention to other possible diagnosis with enlarged corneal diameters to prevent misdiagnosis. The differential diagnosis of anterior megalophthalmos includes megalocornea, congenital glaucoma and keratoglobus. Megalocornea, mostly followed the X-linked recessive inheritance, has no other anomalies similar to anterior megalophthalmos except for corneal enlargement. Keratoglobus is featured with extensive corneal thinning with globular protrusion and high myopia [2, 10-12]. Congenital glaucoma usually presents with elevated IOP, enlarged cornea with Haab's striae and abnormal development of anterior chamber angle [13-16]. Therefore, we could have a more affirmative diagnosis as anterior megalophthalmos in this challenging case after excluding other possibilities.

After diagnosis, another preoperative challenge for anterior megalophthalmos was IOL power calculation. The differences of accuracy between the SRK/T formula and Haigis formula may generate from their respective parameter inclusion. As for SRK/T formula and some other commonly used formulas (Holladay I formula, Hoffer Q formula), the calculation of IOL power is mainly based

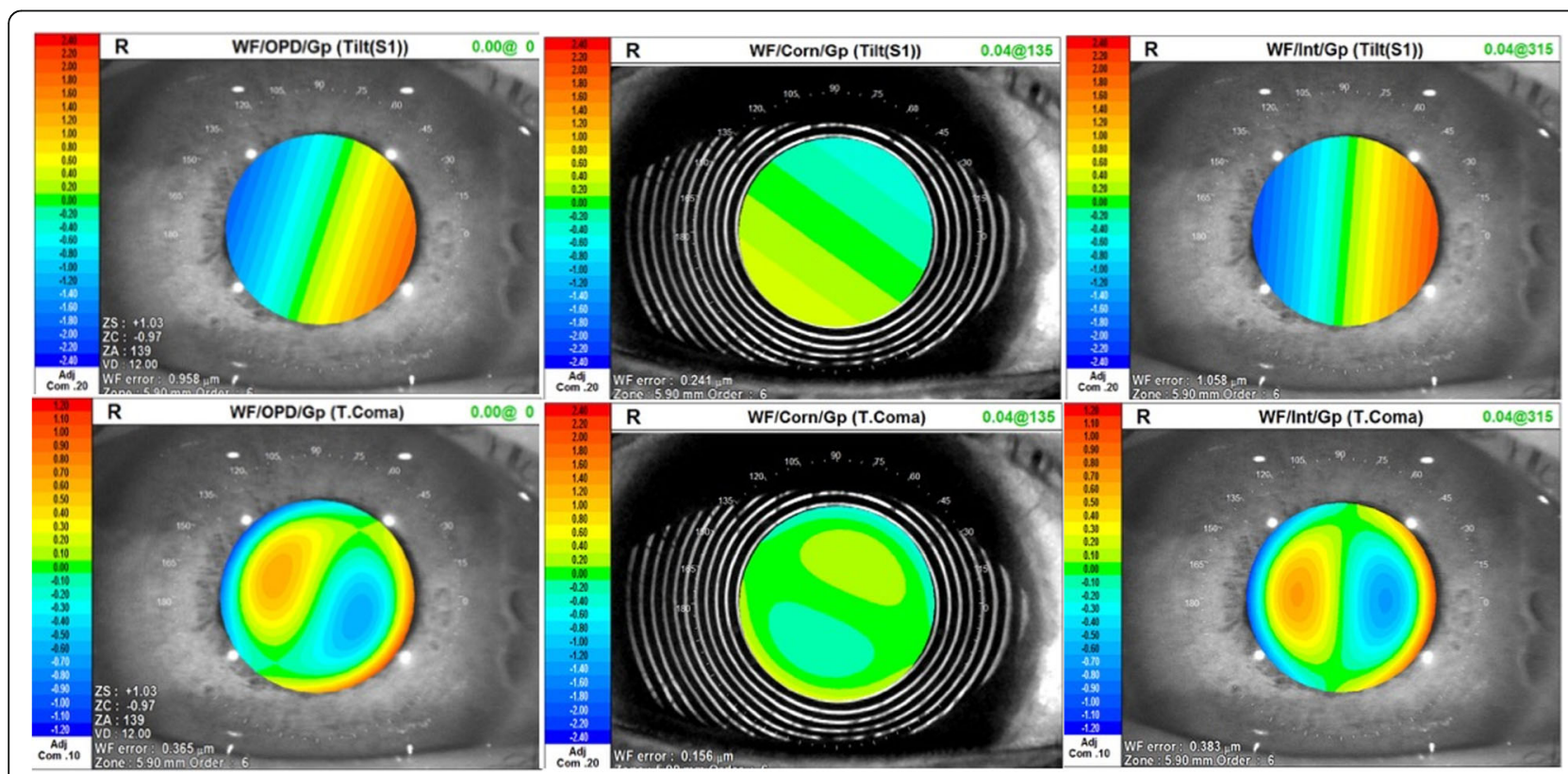

Fig. 4 Postoperative OPD-Scan III aberrometry of the right eye. Main sources of increased coma and tilt aberrations were inside of the eye 
Table 3 Comparisons of different IOL calculation formulas

\begin{tabular}{|c|c|c|c|}
\hline IOL calculation formulas & SRKT & Holladay II & Haigis \\
\hline Predicted refraction (D) & -0.19 & +0.62 & +1.30 \\
\hline Actual postoperative spherical equivalent (D) ${ }^{a}$ & +1.25 & +1.25 & +1.25 \\
\hline $\begin{array}{l}\text { Postoperative refractive error (D) (Postoperative Refractive Error = Actual Postoperative } \\
\text { Spherical Equivalent - Predicted Refraction) }\end{array}$ & +1.44 & +0.63 & -0.05 \\
\hline IOL degree when target diopter equals to $0(D)$ & 20.5 & 21.5 & 22.5 \\
\hline
\end{tabular}

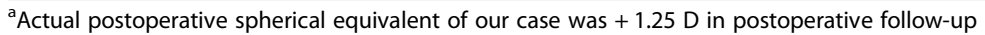

on some given IOL constants, central corneal power and AL $[14,17]$. However, as to the Haigis formula, an additional ocular parameter as ACD is included in the calculation $[15,17]$. For anterior megalophthalmos, one of its symbolic anatomical deformities is deep anterior chambers and taking this parameter into account renders the Haigis formula to be one of the most reliable power formulas as we also proved this in our case. Besides, the Holladay II formula also includes ACD as a calculating parameter [18], but the postoperative refractive error as $+0.63 \mathrm{D}$ in our case, showing a relatively lower suitability than the Haigis formula. Regardless of ACD, corneal curvature is also an important factor in IOL calculation. The corneas of anterior megalophthalmos were reported to be normal $(40.0 \mathrm{D}-45.0 \mathrm{D}, 95 \%$ CI: $42.84 \pm 0.044 \mathrm{D})$ or slightly flatter [7, 19-23]. Our patient also had relatively flat corneas (Table 1). Previous studies showed that the Haigis formula had higher accuracy than the SRK/T formula for $K$ values less than $43.00 \mathrm{D}[24,25]$, which was also confirmed in our case. Therefore, the Haigis formula may be more suitable and accurate for the IOL calculation in anterior megalophthalmos with the smallest postoperative refractive errors. Further study on anterior megalophthalmos with a larger sample size will be needed in the future for evidence-based verification.

The next precarious procedure after IOL power calculation is cataract surgery. Enlarged ACD and anterior chamber width could cause the surgical landmarks and dimensions to be abnormal, which further brings difficulty in estimating capsulorhexis size. Difficulty in preoperative pupil dilation resulted from iridal atrophy, as presented in this case, could make the surgery more challenging for the decrease of operating area. Deficient fixation and traction of capsule can also give rise to common postoperative complications as postoperative IOL dislocation, especially when the IOL implanted is not of proper size or the surgical procedure is not even $[26,27]$.

Negative influences of visual quality brought by IOL dislocation should not be overlooked. Mild or inconspicuous IOL dislocation could presented as monodiplopia and ghosting, indicating impaired visual function. Therefore, confronting this challenge, the cautious selection on the types of IOLs is necessary. We selected MC X11 ASP IOL (HumanOptics AG, Germany), which is lighter than normal with four frame haptics providing strong and firm support within the capsular bag for stable centering. Some recent researches have verified better stability of IOLs with four haptics than those with two haptics. This phenomenon may result from large posterior capsular contact of IOL and constant tension of zonular fibers brought by haptics, which together lead to good IOL centering with less dislocation [28, 29]. Several special IOLs including iris-claw IOL, iris fixation posterior chamber-IOL (PC-IOL) and IOL with stabilizing haptics should be recommended to enhance the stability [30, 31]. Some surgical improvements like suspensory IOL implantation and IOL clamping at capsulorhexis are also helpful for visual rehabilitation [32].

In this case, IOL dislocation with iridal backward concavity still remained on horizontal orientation with flat iris on vertical orientation was observed 1 month after surgery. Unlike previously reports, we firstly applied OPD-Scan III aberrometry in addition to AS-OCT for the evaluation of IOL position, showing a low degree of IOL dislocation as tilt and decentration in the horizontal orientation with increased internal coma aberration in

Table 4 Genes and systemic diseases associated with anterior megalophthalmos and megalocornea

\begin{tabular}{lllll}
\hline Anterior megalophthalmos & & & Megalocornea \\
\cline { 1 - 2 } Gene & Phenotype & & Gene & Phenotype \\
\hline Not Applicable & Trisomy 21 & CHRDL1 & X-linked Megalocornea \\
FBN1 & Marfan's Syndrome & SH3PXD2B & Frank-ter Haar Syndrome \\
FGFR2 & Apert syndrome & NOTCH2 & Hajdu-Cheney Syndrome \\
GNPTAB & Mucolipidosis Type 2 & PIK3R1 & SHORT Syndrome \\
ISPD, B3GNT1, POMT1, POMT2, ISPD, FKTN, FKRP & Walker Warburg Syndrome & ZNF469 & Brittle Cornea Syndrome 1 \\
\hline
\end{tabular}


the right eye. Increased high order aberrations disenable light rays to describe a good retinal image for decreased image contrast and limited range of spatial frequencies, disturbing the further detailed visual processing and visual quality finally $[33,34]$. Owing to the match of inserted IOL in our case, the IOL dislocation was of a low degree, and postoperative visual rehabilitation revealed relatively good.

As a hereditary disease with unclear mechanism, more than half of the previously reported cases are considered to follow X-linked recessive inheritance, while $40 \%$ of the patients inherited autosomally, and only less than $10 \%$ of the patients are idiopathic cases [10]. In this case, the gene analysis based on family by whole-exome sequencing showed no known or newfound diseasecausing gene mutations directly related to anterior megalophthalmos or megalocornea (Table 4) [35-47]. Atypical size of capsule, idiopathic occurrence and unknown pathogenic location are some possible reasons for the gene sequencing result.

We described the clinical manifestation of a challenging anterior megalophthalmos case and the subsequent surgical managements in addition to the follow-up examinations. Our reflection and management of the challenges during the treatment were illustrated in detail. Finally, our patient showed relatively good visual outcomes with a low degree of IOL dislocation. To our knowledge, this is the first case report of anterior megalophthalmos with a comparison of the different IOL power calculation formulas. We also firstly applied the OPD Scan III aberrometry for postoperative examinations on IOL dislocation in this disease. In conclusion, when encountered with challenging anterior megalophthalmos cases, selections of special IOLs should be highlighted to enhance the stability. Although we concluded that the Haigis formula is probably more accurate for the IOL calculation compared to the SRK/T and the Holladay II formulas in this case, it will have to be replicated with a larger sample size in our future study.

\section{Abbreviations}

ACD: Anterior chamber depth; AL: Axial length; AS-OCT: Anterior segment optical coherence tomography; CCT: Central corneal thickness; IOL: Intraocular lens; IOP: Intraocular pressure; LT: Lens thickness; PCIOL: Posterior chamber intraocular lens; SE: Spherical equivalent; UBM: Ultrasound biomicroscopy; WTW: White to white

\section{Acknowlegements}

Not applicable.

\section{Authors' contributions}

AM and KKZ analyzed the data and finished drafting the manuscript. JFY performed a part of statistical analysis and provided technical support. WWH took part in drafting manuscript and statistical analysis; $Y L$ and XJZ gave critical revision of manuscript and were responsible for securing funding, material support and supervision. All authors read and approved the final manuscript.

\section{Funding}

This work was supported by research grants from the National Natural Science Foundation of China (grant nos. 81470613, 81870642, 81670835, 81700819), Shanghai High Myopia Study Group, Shanghai Talent

Development Fund (grant no. 201604), and Outstanding Youth Medical Talents Program of Shanghai Health and Family Planning Commission (grant no. 2017YQ011)

Role of the funding: collection, analysis of data and in writing the manuscript.

Availability of data and materials

All data supporting the findings are contained within the manuscript.

Ethics approval and consent to participate

The study was approved by the Ethics Committee of Ear, Nose, and Throat Hospital of Fudan University (no. 2013021). The ethical standards in the Declaration of Helsinki were adhered to. The written informed consent was obtained from the patient.

\section{Consent for publication}

Written consent for publication was obtained from the patient for publication of this case report and any accompanying images.

\section{Competing interests}

The authors declare that they have no competing interests.

\section{Author details}

${ }^{1}$ Eye Institute, Eye and ENT Hospital, Shanghai Medical College, Fudan University, Shanghai 200031, China. ${ }^{2}$ Department of Ophthalmology and Visual Science, Eye and ENT Hospital, Shanghai Medical College, Fudan University, Shanghai, China. ${ }^{3} \mathrm{NHC}$ Key Laboratory of Myopia (Fudan University), Shanghai, China. ${ }^{4}$ Key Laboratory of Myopia, Chinese Academy of Medical Sciences, Shanghai, China. ${ }^{5}$ Department of Ophthalmology, Beijing Children's Hospital, Capital Medical University, National Center for Children's Health, Beijing, China.

Received: 5 February 2019 Accepted: 22 May 2019

Published online: 30 May 2019

References

1. Neumann AC. Anterior megalophthalmos and intraocular lens implantation. J Am Intraocul Implant Soc. 1984;10(2):220-2.

2. Wright RE. Megalophthalmus And Microphthalmus. Br J Ophthalmol. 1922; 6(1):35-7.

3. Kumawat D, Alam T, Sahay P, Chawla R. Ocular abnormalities and complications in anterior megalophthalmos: a case series. Eye (London, England). 2019;7:1

4. Ahmadieh $H$, Banaee $T$, Javadi MA, Jafarinasab MR, Yazdani S, Sajjadi H. Vitreoretinal disorders in anterior megalophthalmos. Jpn J Ophthalmol. 2006:50(6):515-23.

5. Rao A, Videkar C. Hereditary anterior megalophthalmos with posterior vitreoretinopathy: a surgical challenge. BMJ case reports. 2014: bcr2013202212.

6. Kuchenbecker J, Behrens-Baumann W. Ciliary body dysplasia in megalophthalmos anterior diagnosed using ultrasound biomicroscopy. Eye (London, England). 2002;16(5):638-9.

7. Galvis V, Tello A, MR C. Cataract surgery in anterior Megalophthalmos: a review. Med Hypothesis, Discov Innov Ophthalmol J. 2015;4(3):101-8.

8. Chlasta-Twardzik E, Nowinska A, Was P, Jakubowska A, Wylegala E. Traumatic cataract in patient with anterior megalophthalmos: case report. Medicine. 2017:96(30):e7160

9. Sati A, Murthy SI, Arjun S, Rathi VM. Anterior megalophthalmos: is visual restoration possible? Oman Journal Ophthalmol. 2018;11(2):184-6.

10. Taylor D, Greenwald MJ: Pediatric Ophthalmology: Blackwell Scientific Publications; Chicago, III. : Distributors, USA and Canada, Mosby-Year Book; 1990.

11. Javadi MA, Jafarinasab MR, Mirdehghan SA. Cataract surgery and intraocular lens implantation in anterior megalophthalmos. J Cataract Refract Surg. 2000;26(11):1687-90

12. Gupta N, Ganger A. Keratoglobus: a close entity to megalophthalmos. SpringerPlus. 2016;5:634. 
13. Ho CL, Walton DS. Primary megalocornea: clinical features for differentiation from infantile glaucoma. J Pediatr Ophthalmol Strabismus. 2004:41(1):11-7 quiz 46-17.

14. Retzlaff JA, Sanders DR, Kraff MC. Development of the SRK/T intraocular lens implant power calculation formula. J Cataract Refract Surg. 1990;16(3):333-40.

15. Haigis $\mathbf{W}$. Intraocular lens calculation after refractive surgery for myopia: Haigis-L formula. J Cataract Refract Surg. 2008;34(10):1658-63.

16. Neustein RF, Bruce BB, Beck AD. Primary congenital Glaucoma versus Glaucoma following congenital cataract surgery: comparative clinical features and long-term outcomes. Am J Ophthalmol. 2016;170:214-22.

17. Melles RB, Holladay JT, Chang WJ. Accuracy of intraocular Lens calculation formulas. Ophthalmology. 2018;125(2):169-78.

18. Fenzl RE, Gills JP, Cherchio M. Refractive and visual outcome of hyperopic cataract cases operated on before and after implementation of the Holladay II formula. Ophthalmology. 1998;105(9):1759-64.

19. Matalia JH, Tejwani S, Rajput VK, Matalia H. Small lens for a big eye: successful management of anterior megalophthalmos. Indian J Ophthalmol. 2018;66(3):457-9.

20. Gangwe AB, Bhatia P, Agrawal D, Chatterjee S. Trans-scleral explantation of posteriorly dislocated IOL-CTR complex in a case of anterior megalophthalmos with an unusually thin cornea. BMJ case reports. 2018:bcr-2018.

21. Huang $\mathrm{CX}$, Zhao $\mathrm{XH}$, Xing YQ. Combined phacoemulsification and anterior vitrectomy in a case of anterior megalophthalmos with open-angle glaucoma and high myopia. Int J Ophthalmol. 2017;10(7):1178-80.

22. Jain AK, Nawani N, Singh R. Phacoemulsification in anterior megalophthalmos: rhexis fixation technique for intraocular lens centration. Int Ophthalmol. 2014;34(2):279-84.

23. Guler E, Totan Y, Hepsen IF, Tenlik A. Unilateral spontaneous Descemet membrane rupture in a case with Megalophthalmos. Eye Contact Lens. 2018:44(1):e4-6.

24. Zhu XJ, He WW, Du Y, Qian DJ, Dai JH, Lu Y. Intraocular lens power calculation for high myopic eyes with cataract: comparison of three formulas. Zhonghua Yan Ke Za Zhi [Chin J Ophthalmol]. 2017;53(4):260-5.

25. Abulafia A, Barrett GD, Rotenberg M, Kleinmann G, Levy A, Reitblat O, Koch DD, Wang L, Assia El. Intraocular lens power calculation for eyes with an axial length greater than $26.0 \mathrm{~mm}$ : comparison of formulas and methods. J Cataract Refract Surg. 2015;41(3):548-56.

26. Lee GA, Hann JV, Braga-Mele R. Phacoemulsification in anterior megalophthalmos. J Cataract Refract Surg. 2006;32(7):1081-4.

27. Vaz FM, Osher RH. Cataract surgery and anterior megalophthalmos: custom intraocular lens and special considerations. J Cataract Refract Surg. 2007; 33(12):2147-50.

28. Kretz FT, Breyer D, Klabe K, Auffarth GU, Kaymak H. Clinical outcomes and capsular bag stability of a four-point haptic Bitoric intraocular Lens. J Refractive Surg (Thorofare, NJ : 1995). 2015;31(7):431-6.

29. Kim SY, Yang JW, Lee YC, Kim SY. Effect of haptic material and number of intraocular lens on anterior capsule contraction after cataract surgery. Korean J Ophthalmol: KJO. 2013, 27(1):7-11.

30. Mora P, Calzetti G, Favilla S, Forlini M, Tedesco S, Date P, Tagliavini V, Carta A, Frisina R, Pedrotti $E$, et al. Comparative analysis of the safety and functional outcomes of anterior versus Retropupillary Iris-claw IOL fixation. J Ophthalmol. 2018;2018:8463569.

31. Narvaez J, Nam E. Iris fixation of unstable anterior chamber intraocular lenses. J Cataract Refract Surg. 2016;42(7):961-4.

32. Guixeres Esteve MC, Pardo Saiz AO, Martinez-Costa L, Gonzalez-Ocampo Dorta S, Sanz Solana P. Surgical Management of a Patient with anterior Megalophthalmos, Lens subluxation, and a high risk of retinal detachment. Case Reports Ophthalmol. 2017;8(1):61-6.

33. Marcos S. Image quality of the human eye. Int Ophthalmol Clin. 2003:43(2): $43-62$

34. Amorim-de-Sousa A, Macedo-de-Araujo R, Fernandes P, Queiros A, Gonzalez-Meijome JM. Impact of defocus and high-order aberrations on light disturbance measurements. J Ophthalmol. 2019:2874036.

35. Agrawal P, Patel C, Abidi N, Ramchandani S, Vadhel L, Loomba A. Walker Warburg syndrome associated with microphthalmos and megalophthalmos: a rare combination. Oman J Ophthalmol. 2013;6(2):134-5.

36. Cury GK, Matte U, Artigalas O, Alegra T, Velho RV, Sperb F, Burin MG, Ribeiro EM, Lourenco CM, Kim CA, et al. Mucolipidosis II and III alpha/beta in Brazil: analysis of the GNPTAB gene. Gene. 2013;524(1):59-64.

37. Das S, Munshi A. Research advances in Apert syndrome. J Oral Biol Craniofacial Res. 2018;8(3):194-9.
38. Davidson AE, Cheong SS, Hysi PG, Venturini C, Plagnol V, Ruddle JB, Ali H, Carnt N, Gardner JC, Hassan H, et al. Association of CHRDL1 mutations and variants with $\mathrm{X}$-linked megalocornea, Neuhauser syndrome and central corneal thickness. PLoS One. 2014:9(8):e104163.

39. Desir J, Sznajer Y, Depasse F, Roulez F, Schrooyen M, Meire F, Abramowicz M. LTBP2 null mutations in an autosomal recessive ocular syndrome with megalocornea, spherophakia, and secondary glaucoma. European J Human Genetics : EJHG. 2010;18(7):761-7.

40. Frank Y, Ziprkowski M, Romano A, Stein R, Katznelson MB, Cohen B, Goodman RM. Megalocornea associated with multiple skeletal anomalies: a new genetic syndrome. J de Genetique Humaine. 1973;21(2):67-72.

41. Judge DP, Dietz HC. Marfan's syndrome. Lancet (London, England). 2005; 366(9501):1965-76.

42. Rosser EM, Mann NP, Hall CM, Winter RM. Serpentine fibula syndrome: expansion of the phenotype with three affected siblings. Clin Dysmorphol. 1996;5(2):105-13.

43. Schwingshandl J, Mache CJ, Rath K, Borkenstein MH. SHORT syndrome and insulin resistance. Am J Med Genet. 1993:47(6):907-9.

44. Trkova M, Krutilkova V, Smetanova D, Becvarova V, Hlavova E, Jencikova N, Hodacova J, Hnykova L, Hroncova H, Horacek J, et al. ISPD gene homozygous deletion identified by SNP array confirms prenatal manifestation of Walker-Warburg syndrome. European J Med Genet. 2015; 58(8):372-5

45. Van Reeuwijk J, Olderode-Berends MJ, Van den Elzen C, Brouwer OF, Roscioli T, Van Pampus MG, Scheffer H, Brunner HG, Van Bokhoven $\mathrm{H}, \mathrm{Hol}$ FA. A homozygous FKRP start codon mutation is associated with WalkerWarburg syndrome, the severe end of the clinical spectrum. Clin Genet. 2010;78(3):275-81.

46. Yang H, Manya H, Kobayashi K, Jiao H, Fu X, Xiao J, Li X, Wang J, Jiang Y, Toda $T$, et al. Analysis of phenotype, enzyme activity and genotype of Chinese patients with POMT1 mutation. J Hum Genet. 2016;61(8):753-9.

47. Christensen AE, Knappskog PM, Midtbo M, Gjesdal CG, Mengel-From J, Morling N, Rodahl E, Boman H. Brittle cornea syndrome associated with a missense mutation in the zinc-finger 469 gene. Invest Ophthalmol Vis Sci. 2010;51(1):47-52.

\section{Publisher's Note}

Springer Nature remains neutral with regard to jurisdictional claims in published maps and institutional affiliations.

Ready to submit your research? Choose BMC and benefit from:

- fast, convenient online submission

- thorough peer review by experienced researchers in your field

- rapid publication on acceptance

- support for research data, including large and complex data types

- gold Open Access which fosters wider collaboration and increased citations

- maximum visibility for your research: over $100 \mathrm{M}$ website views per year

At BMC, research is always in progress.

Learn more biomedcentral.com/submission 